\title{
Effect of HPC (Hyper-coal) on Strength of Ferro-coke during Caking Temperature
}

\author{
Ataru UCHIDA, ${ }^{1)}$ Yoshiaki YAMAZAKI, ${ }^{1}$ Shohei MATSUO, ${ }^{11}$ Yasuhiro SAITO, ${ }^{1) *}$ Yohsuke MATSUSHITA, ${ }^{11}$ \\ Hideyuki AOKII and Maki HAMAGUCHI'
}

1) Department of Chemical Engineering, Graduate School of Engineering, Tohoku University 6-6-07 Aoba, Aramaki, Aoba-ku, Sendai, Miyagi, 980-8579 Japan.

2) Kobe Steel, LTD., 2-3-1, Shinhama, Arima-cho, Takasago, Hyogo, 676-8670 Japan.

(Received on March 9, 2017; accepted on April 27, 2017)

\begin{abstract}
The effect of HPC (Hyper-coal), which was affected by the iron oxide, on coal particle adhesion during carbonization was investigated. X-ray diffraction analyses were carried out to obtain changes of iron oxide crystal structure on HPC with iron oxide addition during caking temperature. The promoted weight loss of HPC by the iron oxide during caking temperature was calculated from the weight losses by thermogravimetric analyses and gas concentrations by a gas chromatograph and a Karl Fischer titrator. Furthermore, to reveal the effect of iron oxide on coal particle adhesion at resolidification temperature, the yields of semi-coke with and without HPC/High Fluidity-HPC (HF-HPC)/ $/ \mathrm{Fe}_{2} \mathrm{O}_{3} / \mathrm{Fe}_{3} \mathrm{O}_{4}$ were measured, the strength of the semi-coke samples was evaluated using diametral compression tests, and the adhesion of coal particles was evaluated using microstructure analyses. As a result, the promoted weight loss of HPC was increased by the catalysis of $\mathrm{Fe}_{2} \mathrm{O}_{3}$, and was decreased, when the $\mathrm{Fe}_{2} \mathrm{O}_{3}$ was reduced to $\mathrm{Fe}_{3} \mathrm{O}_{4}$, due to hydrogen atoms in the thermoplastic components of HPC. Thus, the strength of semi-coke with HPC increased. Furthermore, although the iron oxide affected the HPC, the adhesiveness of coal particles, which was an index of ferro-coke strength, was enhanced by HPC, and the adhesiveness of HF-HPC was higher than that of HPC. Therefore, the HPC decreased the chemical effect of iron oxide, and improved the adhesiveness.
\end{abstract}

KEY WORDS: ironmaking; ferro-coke; hyper-coal; coke strength.

\section{Introduction}

Coke plays a role as a reducing agent of iron ore, a heat source, and a spacer in blast furnace operations. ${ }^{1,2)}$ Coke strength and its reactivity are important factors to improve efficiency of blast furnace operations., ${ }^{3,4)}$ Ferro-coke is known as coke with high reactivity, which is produced by adding iron ore to coal in coke-making processes. ${ }^{5,6)}$ Ferrocoke has been investigated for its strength and reactivity. For example, Nomura et al. showed that iron ore decreased expansibility of coal particles though ferro-coke has high reactivity. ${ }^{7)}$ Yamazaki et al. reported that a decrease in expansibility of coal particles caused a decrease in their adhesiveness, and coke strength decreased. ${ }^{8)}$ Therefore, it is necessary to enhance strength of ferro-coke by improvement of adhesiveness of coal particles.

To improve strength of ferro-coke, blending HPC (Hyper-coal) into coal particles in coke-making processes has been investigated.9) Okuyama et al. showed that HPC softened in low temperature and had high fluidity, and demonstrated that blending HPC into low-quality coal contributed the improvement of coke strength. ${ }^{10)}$ Koyano et al. reported that a binder including polycyclic aromatic

\footnotetext{
* Corresponding author: E-mail: saito@tohoku.ac.jp

DOI: http://dx.doi.org/10.2355/isijinternational.ISIJINT-2017-130
}

hydrocarbons which had an affinity for thermoplastic components of coal, improved adhesiveness of coal particles and increased coke strength. ${ }^{11)}$ Moreover, Uchida et al. indicated that blending HPC into coal particles enhanced adhesiveness of coal particles and strength of coke while strength of coke decreased because adhesiveness of coal particles decreased due to the existence of metallic iron around coal particles in microscopic structure of ferro-coke. ${ }^{12}$ These studies evaluated coke samples after carbonization and focused on the relationships between only two components, coal and iron or HPC. To investigate ferro-coke utilized in industrial processes, the evaluation of three components, coal, iron ore, and HPC is required.

Although coke quality after coal carbonization has been evaluated, carbonization process itself is regarded to be important because it is found that iron oxide causes reduction reaction during coal carbonization processes and it affects fluidity of HPC. ${ }^{13)}$ Uchida et al. reported that iron oxide was reduced by hydrogen atoms in HPC and thermoplastic components of coal in thermoplastic temperature, and promoted carbonization of HPC. ${ }^{13)}$ Byambasjav et al. showed that asphaltene was cracked by hematite $\left(\mathrm{Fe}_{2} \mathrm{O}_{3}\right)$ as catalyst at $300^{\circ} \mathrm{C}$, and was decomposed into low molecular compounds. ${ }^{14)}$ This fact suggested that hematite cut $\mathrm{C}-\mathrm{C}$ bond off. However, the effect of reduction reaction and catalysis of iron oxide on coke-making processes has been 
rarely investigated, and in particular, the dominant factors which affect strength of ferro-coke have not been well discussed.

In the present study, the effect of HPC on strength of ferro-coke during caking temperature was investigated. X-ray diffraction analyses for a sample with HPC and iron oxide was performed, and crystal structure changes of iron oxide with reduction reaction were evaluated. To investigate the effect of reduction reaction of iron oxide on weight loss ratio of HPC, weight loss of coal matrix and gas analyses during carbonization were conducted using a thermo gravimeter, a gas chromatograph, and a Karl Fischer titrator. The weight losses of HPC promoted by the iron oxide during caking temperature were estimated from measured and theoretical calculated values. Furthermore, samples with HPC, catalysts, and/or High Fluidity-HPC were employed. Yield ratios of semi-coke samples of ferro-coke were obtained, and the influence of the blending ratio of HPC, kind of catalysts, and fluidity of HPC on their yield ratios was discussed. Moreover, the strength of semi-coke samples of ferro-coke and the adhesiveness of coal particles were evaluated, and dominant factors of ferro-coke strength were discussed.

\section{Experimental}

\subsection{Samples}

Non- or slightly- caking coal was used as a sample, and Hyper-coal (HPC) and High Fluidity-HPC (HF-HPC) were employed as a binder, which are different in fluidity. HF-HPC is derived in higher temperature than HPC, and includes low molecular weight components. Proximate and ultimate analyses of coal, HPC, and HF-HPC used in this experiment are indicated in Table 1. The initial thermoplastic temperature, maximum fluidity, and resolidification temperature of HPC employed in the experiment are summarized in Table 2. HF-HPC shows wide-range thermoplastic temperature, and has higher fluidity than HPC. Hematite $\left(\mathrm{Fe}_{2} \mathrm{O}_{3}\right.$, Wako $\left.95 \%\right)$ and magnetite $\left(\mathrm{Fe}_{3} \mathrm{O}_{4}\right.$, Wako 95\%) were chosen as a catalyst. The diameter and blending ratio of samples are listed in Tables 3 and 4, respectively. Here, in the samples with hematite (i.e., Samples HP00-H, HP05-H, HP10-H, HP15-H, and HfHP05-H) and samples with magnetite (i.e., Samples HP00-M, HP05-M, and HfHP05-M), the amount of hematite and magnetite was determined so as to be equal that of metal iron. The amount of metal iron in samples with $30 \mathrm{wt} \%$ of hematite and 29 $\mathrm{wt} \%$ of magnetite was calculated by following equation:

(Weight of metal iron in sample $)=$

$$
\frac{2 \mathrm{M}_{\mathrm{Fe}}}{\mathrm{M}_{\mathrm{Fe}_{2} \mathrm{O}_{3}}} \times 0.30=\frac{3 \mathrm{M}_{\mathrm{Fe}}}{\mathrm{M}_{\mathrm{Fe}_{3} \mathrm{O}_{4}}} \times 0.29=0.210
$$

where $\mathrm{M}$ is atomic or molecular weight. The difference in weight (i.e., $1 \mathrm{wt} \%$ ) corresponds to oxygen atoms when all the hematite is reduced to magnetite, and is defined as 'Blank' as shown in Table 4. The samples were shaped into tablets by packing and compressing coal in the mold with diameter of $25 \mathrm{~mm}$. Samples HPC100, HPC70-HEM30, and HEM100 were compressed and shaped with pressure of $98 \mathrm{MPa}$ in room temperature. Samples HP00-H,
HP05-H, HP10-H, HP15-H, HP00-M, HP05-M, HfHP05-H, and HfHP05-M were with $294 \mathrm{MPa}$. And then, the tablets of the samples (Samples HP00-H, HP05-H, HP10-H, HP15-H, HP00-M, HP05-M, HfHP05-H, and HfHP05-M) were heated up to $500^{\circ} \mathrm{C}$ with heating rate of $5^{\circ} \mathrm{C} / \mathrm{min}$, and quenched quickly in nitrogen atmosphere. To investigate behaviors of samples during caking temperature, the tablets

Table 1. Characterization of coals.

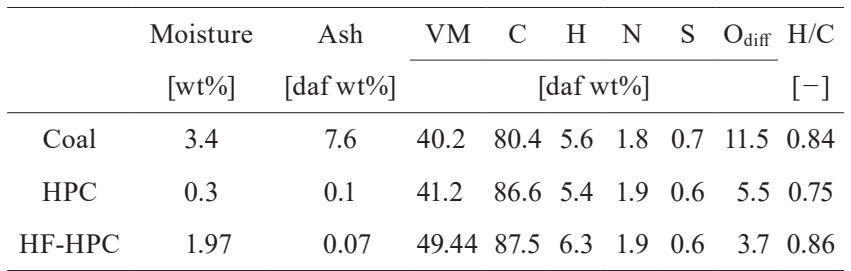

where HPC is hyper-coal, HF-HPC represents high fluidity hyper-coal, and VM is volatile matter.

Table 2. Data of Gieseler plastometry.

\begin{tabular}{lcccc}
\hline & $\begin{array}{c}\text { Initial } \\
\text { softening } \\
\text { temperature } \\
{\left[{ }^{\circ} \mathrm{C}\right]}\end{array}$ & $\begin{array}{c}\text { Maximum } \\
\text { fluidity } \\
\text { temperature } \\
{\left[{ }^{\circ} \mathrm{C}\right]}\end{array}$ & $\begin{array}{c}\text { Resolidification } \\
\text { temperature } \\
{\left[{ }^{\circ} \mathrm{C}\right]}\end{array}$ & $\begin{array}{c}\text { log } \\
(\mathrm{MF} / \mathrm{ddpm})\end{array}$ \\
\hline Coal & 408 & $430-436$ & 451 & 0.78 \\
HPC & 242 & $351-426$ & 476 & $>4.8$ \\
HF-HPC & 177 & $277-448$ & 481 & $>4 / 8$ \\
\hline
\end{tabular}

where HPC is hyper-coal, HF-HPC represents high fluidity hyper-coal, and MF is maximum fluidity.

Table 3. Particle size of raw materials.

\begin{tabular}{lc}
\hline & Diameter \\
\hline $\mathrm{Coal}$ & $<1.0 \mathrm{~mm}$ \\
$\mathrm{HPC}$ (hyper-coal) & $<150 \mu \mathrm{m}$ \\
$\mathrm{HF}-\mathrm{HPC}$ (high fluidity hyper-coal) & $<150 \mu \mathrm{m}$ \\
$\mathrm{Fe}_{2} \mathrm{O}_{3}$ (hematite) & $<150 \mu \mathrm{m}$ \\
$\mathrm{Fe}_{3} \mathrm{O}_{4}$ (magnetite) & $<150 \mu \mathrm{m}$ \\
\hline
\end{tabular}

Table 4. Blending ratio of each sample [wt \%].

\begin{tabular}{lcrcccc}
\hline \multicolumn{1}{c}{ Sample } & Coal & $\mathrm{HPC}$ & $\mathrm{HF}-\mathrm{HPC}$ & $\mathrm{Fe}_{2} \mathrm{O}_{3}$ & $\mathrm{Fe}_{3} \mathrm{O}_{4}$ & Blank \\
\hline HPC100 & 0 & 100 & 0 & 0 & 0 & 0 \\
HPC70-HEM30 & 0 & 70 & 0 & 30 & 0 & 0 \\
HEM100 & 0 & 0 & 0 & 100 & 0 & 0 \\
HP00-H & 70 & 0 & 0 & 30 & 0 & 0 \\
HP05-H & 65 & 5 & 0 & 30 & 0 & 0 \\
HP10-H & 60 & 10 & 0 & 30 & 0 & 0 \\
HP15-H & 55 & 15 & 0 & 30 & 0 & 0 \\
HP00-M & 70 & 0 & 0 & 0 & 29 & 1 \\
HP05-M & 65 & 5 & 0 & 0 & 29 & 1 \\
HfHP05-H & 65 & 0 & 5 & 30 & 0 & 0 \\
HfHP05-M & 65 & 0 & 5 & 0 & 29 & 1 \\
\hline
\end{tabular}

where HPC is hyper-coal, and HF-HPC represents high fluidity hyper-coal. $\mathrm{Fe}_{2} \mathrm{O}_{3}$ and $\mathrm{Fe}_{3} \mathrm{O}_{4}$ mean hematite and magnetite, respectively. 
at carbonization temperature of $500^{\circ} \mathrm{C}$ were employed for the evaluation of yield, diametral-compression tests, and microscopic structure analyses. Because the resolidification temperatures of coal, HPC, and HF-HPC are less than $500^{\circ} \mathrm{C}$, the state of the tablets at carbonization temperature of $500^{\circ} \mathrm{C}$ is just the beginning of resolidification.

\subsection{XRD Analysis}

To investigate crystal structure change of iron oxide with reduction reaction during carbonization process, the structure of iron oxide in the Sample HPC70-HEM30 was analyzed at carbonization temperatures of 300, 400, 500, 600,700 , and $1000^{\circ} \mathrm{C}$ by using X-ray diffractometer, XRD (RINT1500, Rigaku Corp.).

\subsection{Thermogravimetric Analysis}

To investigate the effect of reduction reaction of iron oxide on weight loss of coal matrix during carbonization, weight loss of samples was measured using a thermo gravimeter (TG/DTA 220, Seiko Instruments, Inc.). The Samples HPC100, HPC70-HEM30, and HEM100 of about $1 \mathrm{mg}$ were heated up to $1000^{\circ} \mathrm{C}$ with heating rate of $5^{\circ} \mathrm{C} / \mathrm{min}$ under nitrogen atmosphere. The Sample HPC70-HEM30 is mixture of $70 \mathrm{wt} \%$ of HPC and $30 \mathrm{wt} \%$ of hematite, and if the reduction reaction of iron oxide does not affect coal matrix, weight loss of the mixture can be theoretically represented as following equation:

$$
W_{\text {calc }}=0.7 \times W_{\mathrm{HPC} 100}+0.3 \times W_{\mathrm{HEM} 100} .
$$

\subsection{Gas Analysis}

Light gas concentrations $\left(\mathrm{H}_{2}, \mathrm{CH}_{4}, \mathrm{H}_{2} \mathrm{O}, \mathrm{CO}\right.$, and $\left.\mathrm{CO}_{2}\right)$ released from the tablets of the Samples HPC100 and HPC70-HEM30 (about $1 \mathrm{mg}$ ) during carbonization were measured to investigate the components of weight loss ratio of the samples. Crystal water released from pure iron oxide was measured by the weight loss of samples. Gas concentration of $\mathrm{H}_{2}, \mathrm{CH}_{4}, \mathrm{CO}$, and $\mathrm{CO}_{2}$ were measured by 490-GC (Varian), and that of $\mathrm{H}_{2} \mathrm{O}$ by Karl Fischer titrator (Mitsubishi Chemical Analytech Co. Ltd.), respectively.

\subsection{Evaluation of Yield}

Yield ratios were evaluated from difference in weight of samples before and after carbonization, and calculated by Eq. (3):

$$
(\text { Yield ratio })=\frac{Y}{Y_{0}+Y_{\text {blank }}} \times 100,
$$

where $Y_{0}$ and $Y$ are weight of the samples before and after carbonization. $Y_{\text {blank }}$ is difference in weight of hematite and magnetite added to the samples, and in the case of Samples HP00-H, HP05-H, HP10-H, HP15-H, and HfHP05-H, the value is zero.

\subsection{Diametral-compression Test}

Diametral-compression tests were performed for coke samples using a universal tester (Shimadzu Corp., Autograph AG-150kN). Load was put on a sample with loading rate of $2 \mathrm{~mm} / \mathrm{min}$. The number of tests for each sample was from seven to eight times. Scale parameter was calculated using the Weibull plot in the same way of Sakai et al. ${ }^{15)}$ The calculation method was the same method as that employed by Kanai et $a l .{ }^{16)}$ Here, the scale parameter means tensile strength, and can evaluate strength of semi-coke sample.

\subsection{Microscopic Structure Analysis}

Epoxy resin was embedded in the Samples HP00-H, HP05-H, HP10-H, HP15-H, HP00-M, HP05-M, HfHP05-H, and HfHP05-M with vacuum impregnation equipment, and the samples were polished. Digital images of polished surface were obtained by using optical microscope (LV-100-POL, Nikon) for the above samples. The image size was $3.11 \mathrm{~mm} \times 2.33 \mathrm{~mm}$, and the display resolution was 1280 pixel $\times 960$ pixel. Thirty images were taken for each sample, and were combined and trimmed to obtain the combined image with size of $10 \mathrm{~mm} \times 10 \mathrm{~mm}$ and display resolution of 4116 pixels $\times 4116$ pixels. Pores were extracted by setting threshold for brightness of pixel in digital microscopic image and binarizing the image with discriminant analysis method using image analysis software (WinROOF ver. 6.1, Mitani Corp.). Roundness of pores was obtained by analyzing combined binary image with the image analysis software. Roundness is a shape parameter, and is defined as follows:

$$
(\text { Roundness })=\frac{4 \pi S}{L^{2}},
$$

where $L$ and $S$ are perimeter and area of pore, respectively.

\section{Results and Discussion}

\subsection{Changes of Crystal Structure of Iron Oxide}

To investigate changes of crystal structure of iron oxide in carbonization process, the crystal structure was analyzed using XRD. The measured results in the Sample HPC70-HEM30 at carbonization temperatures from 300 to $1000^{\circ} \mathrm{C}$ are shown in Fig. 1. Iron oxide was reduced from hematite to magnetite with an increase in temperature at $300-500^{\circ} \mathrm{C}$. Hematite seems to be reduced to magnetite by hydrogen atom in softened HPC at $300-500^{\circ} \mathrm{C}$ in terms of the previous study. ${ }^{13)}$ Fraction of magnetite increased at $500-600^{\circ} \mathrm{C}$, and magnetite was reduced to wustite $(\mathrm{FeO})$ or metal iron $(\mathrm{Fe})$ over $700^{\circ} \mathrm{C}$. The above results mean that the crystal structure of iron oxide changed with reduction reaction of iron oxide in carbonization process. The effect

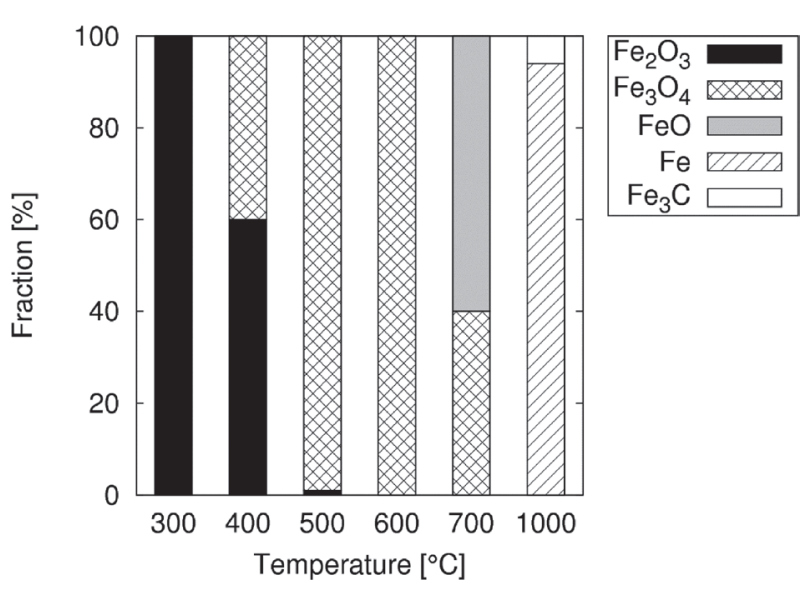

Fig. 1. Iron oxide transitions of Sample HPC70-HEM30. 
of iron oxide on thermoplastic components of HPC would change with changes in crystal structure of iron oxide because reduction reaction of iron oxide was promoted by hydrogen in softened components of HPC in thermoplastic state. ${ }^{13)}$ From the above results, weight loss ratio of thermoplastic components of HPC would change and weight of HPC contributing adhesiveness of coal particles would also change because reduction reaction of iron oxide from hematite to magnetite is promoted in $300-500^{\circ} \mathrm{C}$.

\subsection{Effect of Reduction of Iron Oxide on Weight Loss Ratio of HPC}

Thermogravimetric analyses were carried out to investigate weight loss ratio of HPC promoted with change in crystal structure of iron oxide in carbonization process. The results of thermogravimetric analyses are shown in Fig. 2. The solid line is the measured value of Sample HPC70-HEM30, and dashed line is theoretically calculated one from the measured values of Samples HPC100 and HEM100 as the ratio of 7:3 as shown in Eq. (2). The measured value of Sample HPC70-HEM30 (i.e., with interaction) involves weight loss ratio of HPC promoted by reduction reaction of iron oxide, that of oxygen atoms abstracted from iron oxide reagent with reduction reaction of iron oxide, and crystal water originally in iron oxide reagent. On the other hand, the calculated one (i.e., without interaction) does not involve weight loss ratio of HPC promoted by reduction reaction of iron oxide and that of oxygen atoms abstracted from iron oxide reagent with reduction reaction of iron oxide. In the present study, weight loss ratio of HPC promoted by reduction reaction of iron oxide in the Sample HPC70-HEM30 was estimated by following method.

Figure 3 shows weight loss ratios of Sample HPC70-HEM30 and those calculated by weight loss ratio of Samples HPC100 and HEM100, which were measured individually. Here, the weight loss ratios, $W$ were normalized based on the initial weight of each chemical species. The difference of $W_{\text {HPC70-HEM30 }}$ (Fig. 3(a)) and $W_{\text {calc }}$ (Fig. 3(b)) is regarded as weight loss ratio of sample, which is promoted by reduction reaction of iron oxide as follows:

$$
\Delta W_{\text {promoted,total }}=W_{\mathrm{HPC} 70-\mathrm{HEM} 30}-W_{\text {calc }} .
$$

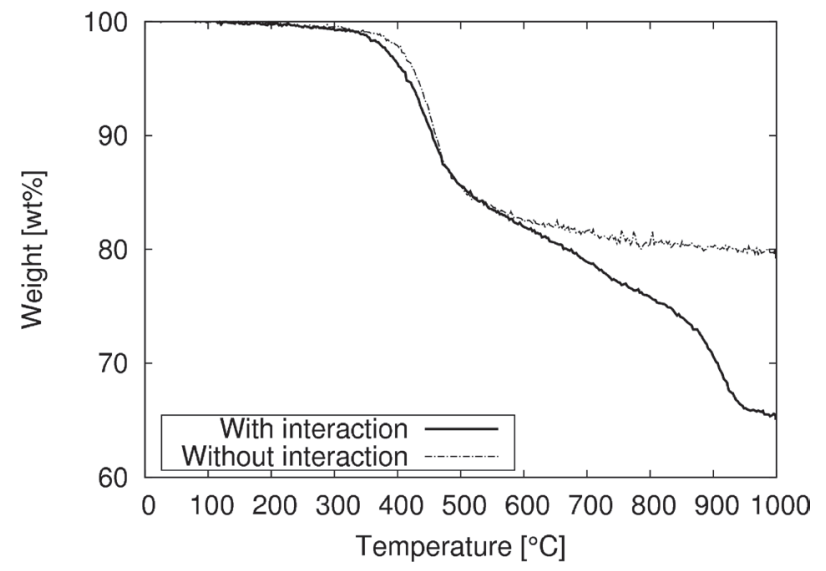

Fig. 2. Weight loss of sample (HPC: $\mathrm{Fe}_{2} \mathrm{O}_{3}=70: 30$ ). "With interaction" indicates the measured value of Sample HPC70-HEM30, and "Without interaction" indicates theoretically calculated one from the measured values of Samples HPC100 and HEM100.
Furthermore, in the present experimental condition, the experiments were performed in nitrogen atmosphere, and the measured value of Sample HPC70-HEM30 and the calculated one from the measured values of Sample HPC100 and HEM100 were compared. Thus, we can evaluate the interaction of HPC and oxygen atoms abstracted from iron oxide reagent with reduction reaction of iron oxide. The weight loss ratio of HPC caused by reduction reaction of iron oxide, $\Delta W_{\text {promoted,HPC }}$ is obtained by subtracting from $\Delta W_{\text {promoted,total }}$ to $\Delta W_{\text {promoted, }}$ as following equation:

$$
\Delta W_{\text {promoted,total }}=\Delta W_{\text {promoted, } \mathrm{HPC}}+\Delta W_{\text {promoted }, \mathrm{O},} \cdot
$$


involves weight loss ratio of gases with oxygen atom $(\mathrm{CO}$, $\mathrm{CO}_{2}$, and $\mathrm{H}_{2} \mathrm{O}$ ). Figure 4 shows the weight loss ratios of all the released gas components measured by the gas chromatograph and Karl Fischer titrator. In the present study, those gas components were divided into light gas components $\left(\mathrm{H}_{2}, \mathrm{CH}_{4}, \mathrm{H}_{2} \mathrm{O}, \mathrm{CO}\right.$, and $\left.\mathrm{CO}_{2}\right)$ and the other, and the low molecular weight gases were classified into each atom, such as $\mathrm{C}, \mathrm{H}$, and $\mathrm{O}$ shown in Fig. 4(b). The weight loss ratios of $\Delta W_{\text {promoted,o }}$ and $\Delta W_{\text {promoted,total were obtained. Here, }}$ $\Delta W_{\text {promoted,o }}$ was calculated from Eq. (7):

$$
\begin{aligned}
\Delta W_{\text {promoted }, \mathrm{O}} & =\frac{\mathrm{M}_{\mathrm{O}}}{\mathrm{M}_{\mathrm{CO}}} \Delta W_{\text {promoted, } \mathrm{CO}}+\frac{2 \mathrm{M}_{\mathrm{O}}}{\mathrm{M}_{\mathrm{CO}_{2}}} \Delta W_{\text {promoted, } \mathrm{CO}} \\
& +\frac{\mathrm{M}_{\mathrm{O}}}{\mathrm{M}_{\mathrm{H}_{2} \mathrm{O}}} \Delta W_{\text {promoted, } \mathrm{H}_{2} \mathrm{O}},
\end{aligned}
$$

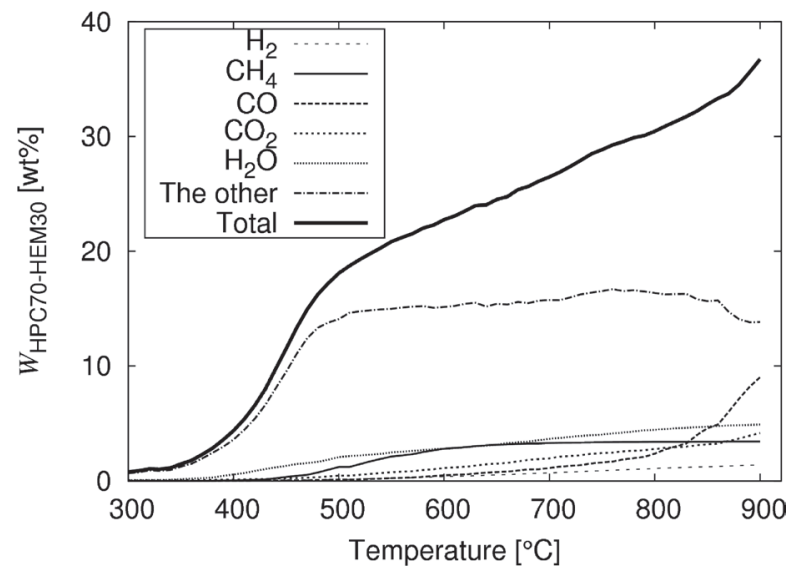

(a) Measured value of Sample HPC70-HEM30

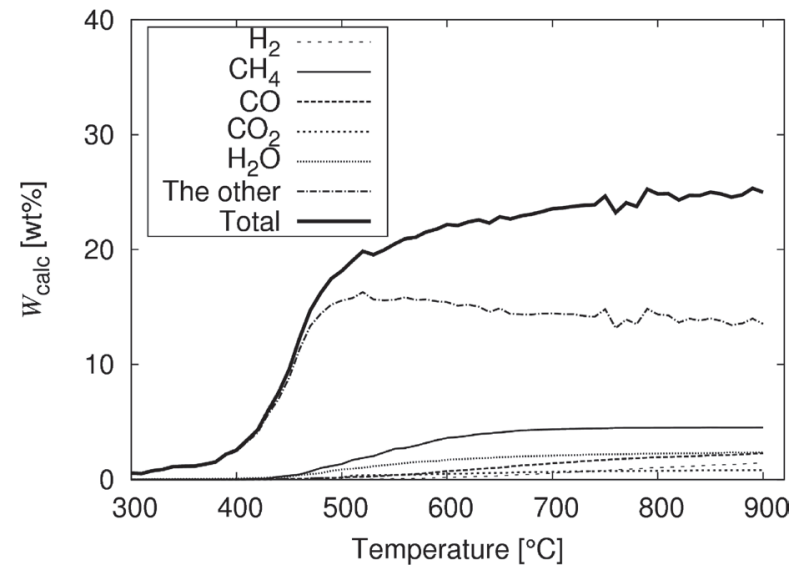

(b) Calculated value from the measured values of Samples HPC100 and HEM100

Fig. 3. Relative weight loss ratio of sample ( $\mathrm{HPC}: \mathrm{Fe}_{2} \mathrm{O}_{3}=70$ : $30)$. 
where $\mathrm{M}$ is atomic or molecular weight. Therefore, the weight loss ratio of HPC, $\Delta W_{\text {promoted,HPC which is caused }}$ by reduction reaction of iron oxide can be estimated by subtracting that of $\Delta W_{\text {promoted,o }}$ from $\Delta W_{\text {promoted,total, and }}$ Fig. 5 was derived from Figs. 3 and 4. For example, at temperature of $500^{\circ} \mathrm{C}$, the weight loss ratios were $\Delta W_{\text {promoted,CO }}=-0.0525 \mathrm{wt} \%, \Delta W_{\text {promoted, } \mathrm{CO}_{2}}=0.287 \mathrm{wt} \%$, $\Delta W_{\text {promoted, } \mathrm{H}_{2} \mathrm{O}}=1.194 \mathrm{wt} \%$, and $\Delta W_{\text {promoted,total }}=-0.0590 \mathrm{wt} \%$. Thus, because $\Delta W_{\text {promoted, } \mathrm{O}}=1.241 \mathrm{wt} \%, \Delta W_{\text {promoted,HPC }}=$ $-1.30 \mathrm{wt} \%$.

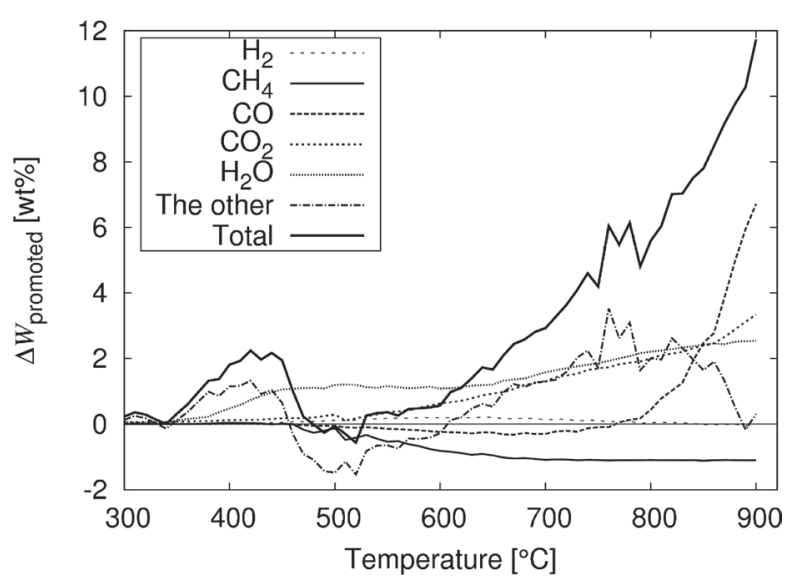

(a) All the released gas components in Sample HPC70-HEM30

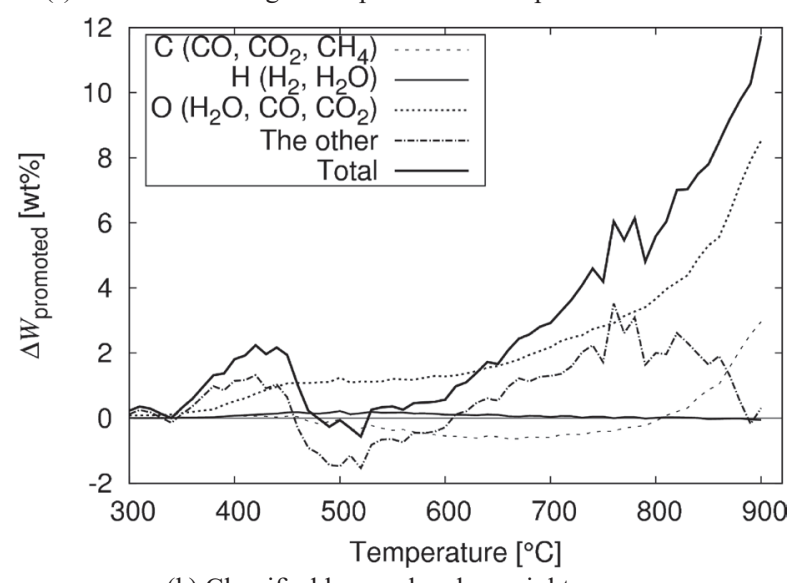

(b) Classified low molecular weight gases

Fig. 4. Relative weight loss of gas components in Sample HPC70HME30 (HPC: $\mathrm{Fe}_{2} \mathrm{O}_{3}=70: 30$ ).

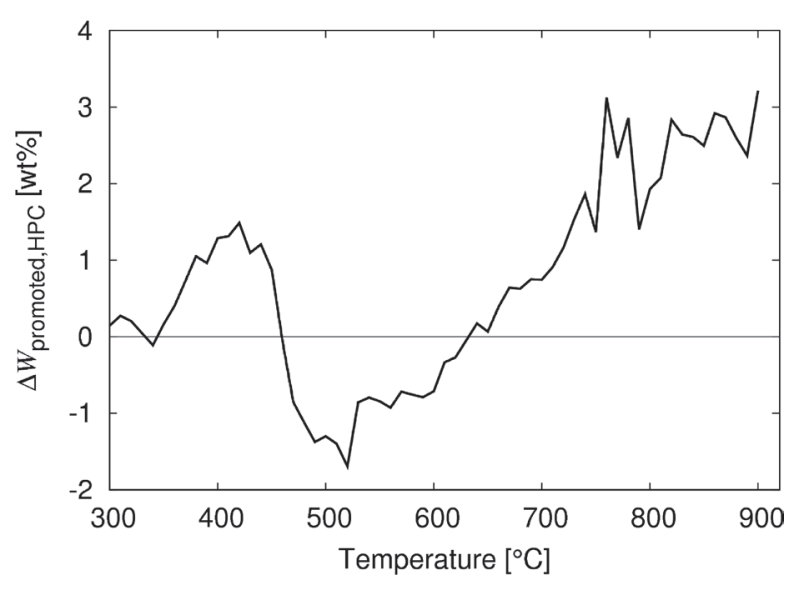

Fig. 5. Promoted relative weight loss of HPC by reduction reaction of iron oxide (HPC: $\mathrm{Fe}_{2} \mathrm{O}_{3}=70: 30$ ).
Figure 5 shows $\Delta W_{\text {promoted,HPC }}$ which is promoted by reduction reaction of iron oxide. The amount of weight loss of thermoplastic components of HPC ( $\left.\Delta W_{\text {promoted,HPC }}\right)$ increased at $300-450^{\circ} \mathrm{C}$. This is because hematite is thought to cut $\mathrm{C}-\mathrm{C}$ bond of hydrocarbon in thermoplastic HPC and to contribute to release of thermoplastic components. ${ }^{14)}$ Weight loss ratio of thermoplastic components in HPC decreased at $460-630^{\circ} \mathrm{C}$. In other words, carbonization of HPC was promoted in this temperature range, and the release of thermoplastic components was inhibited. From the fact that the release of hydrogen was promoted, this is caused by the reduction of hematite with hydrogen in thermoplastic HPC because the hydrogen reduction of iron oxide occurred. Furthermore, dehydrogenation of thermoplastic components of HPC occurred because the release of gases with carbon atoms and the other gases was inhibited, and the carbonization of HPC would be promoted.

\subsection{Effect of Blending Ratio of HPC, Kind of Cata- lysts, and Fluidity of HPC on Yield Ratio}

Yield ratio of semi-coke samples at temperature of $500^{\circ} \mathrm{C}$ is obtained to investigate the effect of blending ratio of $\mathrm{HPC}$, catalyst, and fluidity of HPC on yield ratio of semi-coke in thermoplastic temperature. Yield ratios of the Samples HP00-H, HP05-H, HP10-H, HP15-H, HP00-M, HP05-M, HfHP05-H, and HfHP05-M are shown in Fig. 6. Here, the yield ratios were calculated from Eq. (3). Focusing on the Sample HP00-H ((i) in Fig. 6), HP05-H ((ii) in Fig. 6), HP10-H ((iii) in Fig. 6), and HP15-H ((iv) in Fig. 6), the yield ratio increased with an increase in blending ratio of HPC. This would be because weight loss with reduction reaction of iron oxide at $500^{\circ} \mathrm{C}$ was suspended, and carbonization of HPC was promoted. Moreover, comparing yield ratios of samples with hematite (i.e., Samples HP00-H ((i) in Fig. 6) and HP05-H ((ii) in Fig. 6)) and ones with magnetite (i.e., Samples HP00-M ((v) in Fig. 6) and HP05-M ((vi) in Fig. 6)), yield ratio was lower in the samples with magnetite than in those with hematite. This is because although reduction reaction from hematite to magnetite occurred at $500^{\circ} \mathrm{C}$ as shown in Fig. 1, the reaction from magnetite to hematite did not occur, and carbonization of thermoplastic



Fig. 6. Difference of yield ratio of each sample at carbonization temperature of $500^{\circ} \mathrm{C}$ : (i) Sample HP00-H; (ii) Sample HP05-H; (iii) Sample HP10-H; (iv) Sample HP15-H; (v) Sample HP00-M; (vi) Sample HP05-M; (vii) Sample HfHP05-H; (viii) Sample HfHP05-M. 
components in coal and HPC was not promoted. In addition, large difference did not appear in yield ratio when the same kind of iron oxide was used, in other words, when the Sample HP05-H ((ii) in Fig. 6) is compared with the Sample HfHP05-H ((vii) in Fig. 6), and the Sample HP05-M ((vi) in Fig. 6) with the Sample HfHP05-M ((viii) in Fig. 6). Therefore, HPC increased yield ratio independent of its fluidity. Moreover, when HF-HPC was employed as a binder instead of HPC, yield ratio of the Sample HfHP05-H ((vii) in Fig. 6) was higher than that of the Sample HfHP05-M ((viii) in Fig. 6) as is the case with HPC. It is shown that carbonization of HPC is promoted by reduction reaction of hematite independent of its fluidity.

\subsection{Adhesiveness of Coal Particles in Semi-coke}

According to above results, yield ratio of ferro-coke with HPC is found to increase in thermoplastic temperature because reduction reaction from hematite to magnetite by hydrogen atoms of HPC is promoted. It seems that HPC increases thermoplastic components of HPC and coal which contribute to adhesiveness of coal particles. Therefore, adhesiveness of coal particles, which is dominant factor of strength of ferro-coke seems to be formed with being influenced by iron oxide. Hence, strength of semi-coke and adhesiveness of coal particles in resolidification temperature after thermoplastic temperature are investigated.

Weibull plots of the Samples HP00-H, HP05-H, HP10-H, HP15-H, HP00-M, HP05-M, HfHP05-H, and HfHP05-M obtained by diametral compression tests, and the scale parameters calculated from the Weibull plots are shown in Fig. 7. Furthermore, Fig. 8 shows existence ratio of roundness of pores in the Sample HP00-H, HP05-H, HP00-M, HP05-M, HfHP05-H, and HfHP05-M. Comparing samples with coal and hematite and those with coal and magnetite, the scale parameter of Sample HP00-H ((i) in Fig. 7) is smaller than that of Sample HP00-M ((v) in Fig. 7). This would indicate that thermoplastic components of coal were decomposed due to catalysis of hematite, and the adhesiveness of coal particles decreased. In the previous study, Uchida et al. ${ }^{12)}$ defined pores with roundness of less than 0.05 as non-adhesion pores with iron particles between coal particles in experiments with ferro-coke combined with HPC, and reported that adhesiveness of coal particles can be evaluated by finding the pores with roundness of less than 0.05 . In the present study, the existence ratio of pores with roundness of less than 0.05 was used as index of the adhesiveness.

Focusing on Samples HP00-H ((i) in Fig. 7), HP05-H ((ii) in Fig. 7), HP10-H ((iii) in Fig. 7), and HP15-H ((iv) in Fig. 7), the scale parameter increased with the addition of HPC. Therefore, adhesiveness of coal particles would be enhanced with the addition of HPC. This is derived from the fact that the existence ratio of pores with low roundness of less than 0.05 in the Sample HP05-H ((ii) in Fig. 8) was lower than that in the Sample HP00-H ((i) in Fig. 8). Furthermore, comparing sample with coal, HPC, and hematite (i.e., Sample HP05-H) and the one with coal, HPC, and magnetite (i.e., Sample HP05-M), scale parameter of Sample HP05-M ((vi) in Fig. 7) is larger than that of Sample HP05-H ((ii) in Fig. 7). It is shown that adhesiveness of coal particles increased due to the addition of HPC because the



(a) Weibull plot for each sample

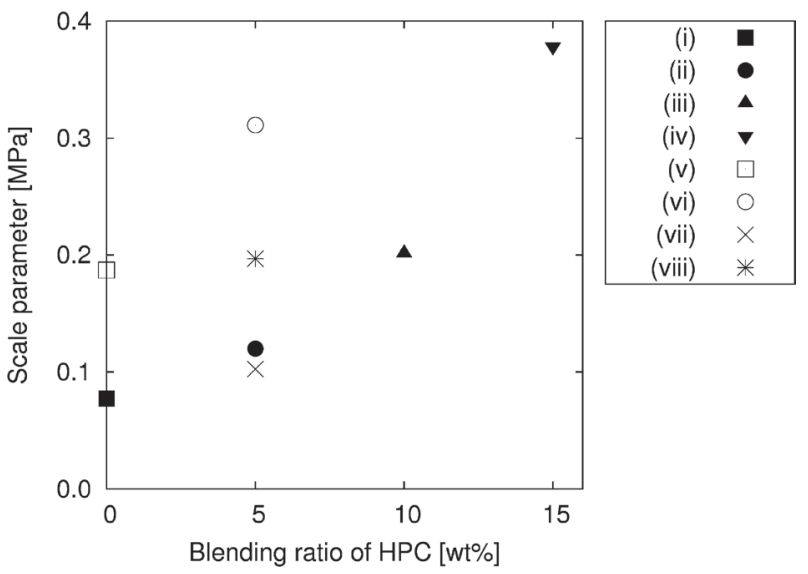

(b) Relationship between material and scale parameter

Fig. 7. Coke strength for each sample at carbonization temperature of $500^{\circ} \mathrm{C}$ : (i) Sample HP00-H; (ii) Sample HP05-H; (iii) Sample HP10-H; (iv) Sample HP15-H; (v) Sample HP00-M; (vi) Sample HP05-M; (vii) Sample HfHP05-H; (viii) Sample HfHP05-M.



Fig. 8. Distributions of pore roundness of each sample at carbonization temperature of $500^{\circ} \mathrm{C}$ : (i) Sample HP00-H; (ii) Sample HP05-H; (v) Sample HP00-M; (vi) Sample HP05M; (vii) Sample HfHP05-H; (viii) Sample HfHP05-M.

existence ratio of pores with low roundness of less than 0.05 in Sample HP05-M ((vi) in Fig. 8) was lower than that in Sample HP05-H ((ii) in Fig. 8).

The Samples HP05-H, HP05-M, HfHP05-H, and HfHP05-M are compared to investigate effect of difference in fluidity of HPC in thermoplastic temperature on adhesiveness of coal particles. For the same catalyst, the 
scale parameters of the Samples HfHP05-H ((vii) in Fig. 7) and HfHP05-M ((viii) in Fig. 7) indicate lower in strength than those of Samples HP05-H ((ii) in Fig. 7) and HP05-M ((vi) in Fig. 7). Yamamoto et al. reported that strength of ferro-coke carbonized in high temperature was increased by increasing blending ratio of a binder and enhancing fluidity of blended sample. ${ }^{17}$ The samples with HF-HPC did not carbonize at $500^{\circ} \mathrm{C}$ more than that with $\mathrm{HPC}$ because resolidification temperature of HF-HPC is higher than that of HPC. Therefore, the strength of the Samples HP05-H ((ii) in Fig. 7) and HP05-M ((vi) in Fig. 7) was higher than that of the Samples HfHP05-H ((vii) in Fig. 7) and HfHP05-M ((viii) in Fig. 7) because the samples with HPC were resolidificated at lower temperature than those with HF-HPC, and the carbonization of the samples with HPC progressed more than those with HF-HPC. Adhesiveness of the samples with HF-HPC seems to be higher than that with HPC because the existence ratio of pores with roundness of less than 0.05 in the Sample HfHP05-H ((vii) in Fig. 8) was less than that in the Sample HP05-H ((ii) in Fig. 8). In the semi-coke samples, HF-HPC flows into space between coal particles and enhances adhesiveness of coal particles in thermoplastic temperature because HF-HPC has higher fluidity than HPC. Therefore, HF-HPC has possibility to improve coke strength compared with HPC if carbonization of the sample with HF-HPC can progress.

From above results, HPC influenced by iron oxide in thermoplastic temperature softens and flows into space between coal particles, and enhances adhesiveness of coal particles. Consequently, dominant factors of ferro-coke strength are found to be formed by thermoplastic components of coal and HPC.

\section{Conclusions}

In the present study, to investigate Hyper-coal (HPC) influenced by iron oxide in thermoplastic process on microscopic structure of ferro-coke, HPC and hematite $\left(\mathrm{Fe}_{3} \mathrm{O}_{4}\right)$ as well as High Fluidity-HPC (HF-HPC) and magnetite $\left(\mathrm{Fe}_{2} \mathrm{O}_{3}\right)$ were employed, and thermogravimetry, X-ray diffraction (XRD) analyses, diametral compression tests, and structure analyses with microscopic image were performed. This study can be summarized as follows:

(1) Hematite was reduced to magnetite by hydrogen atoms of thermoplastic components of coal or HPC at 300$500^{\circ} \mathrm{C}$. The reduction reaction from hematite to magnetite promoted carbonization of HPC, and yield ratio of HPC at resolidification increased.

(2) Thermoplastic components of coal were decomposed by catalysis of hematite at $300-450^{\circ} \mathrm{C}$.

(3) An addition of HPC increased yield ratio of samples, and enhanced adhesiveness of coal particles at resolidification. HF-HPC would improve adhesiveness of semi-coke more than HPC.

Above the results, it is necessary to improve adhesiveness of coal particles with HPC which is rich in hydrogen and softens at low temperature to enhance strength of ferro-coke.

\section{Acknowledgements}

We would like to express our sincere appreciation to the authors for their permission to use some of the results found in "Elucidation of the Mechanism for Developing Strength of New Binders." This study was carried out on consignment from Kobe Steel, Ltd. as a part of "Project to Develop Technology for an innovative Iron-making Process for Efficient Use of Resources," which was funded by the New Energy and Industrial Technology Development Organization (NEDO).

\section{Nomenclature}

$\begin{array}{cc}L: \text { perimeter of pore } & {[\mathrm{m}]} \\ \mathrm{M}: \text { atomic or molecular weight } & {[\mathrm{g} / \mathrm{mol}]} \\ S: \text { area of pore } & {\left[\mathrm{m}^{2}\right]} \\ W: \text { weight loss ratio } & {[\mathrm{wt} \%]} \\ \Delta W: \text { weight loss ratio } & {[\mathrm{wt} \%]} \\ Y: \text { weight of sample } & {[\mathrm{g}]}\end{array}$

Subscripts

blank: difference in weight of hematite and magnetite

HPC: HPC

calc: theoretical calculated

O: oxygen

promoted: promoted

HPC100: Sample HPC100

HPC70-HEM30: Sample HPC70-HEM30

HEM100: Sample HEM100

total: total

0: initial

\section{REFERENCES}

1) I. Shimoyama: Tetsu-to-Hagané, 96 (2010), 209.

2) M. Shimizu and M. Naito: Tetsu-to-Hagané, 92 (2006), 694.

3) M. Naito, A. Okamoto, K. Yamaguchi, T. Yamaguichi and Y. Inoue: Tetsu-to-Hagané, 87 (2001), 357.

4) K. Higuchi, S. Nomura, K. Kunimoto, H. Yokoyama and M. Naito: Tetsu-to-Hagané, 98 (2012), 517.

5) M. Naito, S. Nomura and K. Kato: Tetsu-to-Hagané, 96 (2010), 201.

6) T. Yamamoto, T. Sato, H. Fujimoto, T. Anyashiki, K. Fukuda, M. Sato, K. Takeda and T. Ariyama: Tetsu-to-Hagané, 97 (2011), 501.

7) S. Nomura, H. Terashima, E. Sato and M. Naito: Tetsu-to-Hagané, 92 (2006), 849.

8) Y. Yamazaki, H. Hayashizaki, K. Ueoka, K. Hiraki, Y. Matsushita, H. Aoki and T. Miura: Tetsu-to-Hagané, 96 (2010), 536.

9) T. Takanohashi, T. Shishido, H. Kawashima and I. Sato: Fuel, 87 (2008), 592.

10) N. Okuyama, T. Shigehisa, Y. Nishibata, K. Matsudaira and M. Nishimura: Tetsu-to-Hagané, 92 (2006), 213.

11) K. Koyano, K. Ueoka, T. Takanohashi, K. Fukuda and K. Ota: ISIJ Int., 51 (2011), 1044.

12) A. Uchida, T. Kanai, Y. Yamazaki, K. Hiraki, Y. Saito, H. Aoki, N. Komatsu, N. Okuyama and M. Hamaguchi: ISIJ Int., 53 (2013), 403.

13) A. Uchida, Y. Yamazaki, S. Matsuo, Y. Saito, H. Aoki and M. Hamaguchi: ISIJ Int., 56 (2016), 2132.

14) E. Byambajav and Y. Ohtsuka: Appl. Catal. A: Gen., 252 (2003), 193.

15) M. Sakai, R. Nishimura, M. Nishimura and K. Fukuda: Tetsu-toHagané, 92 (2006), 164

16) T. Kanai, Y. Yamazaki, X. Zhang, A. Uchida, Y. Saito, M. Shoji, H. Aoki, S. Nomura, Y. Kubota, H. Hayashizaki and S. Miyashita: $J$. Therm. Sci. Technol., 7 (2012), 351.

17) T. Yamamoto, K. Sato, H. Fujimoto, T. Anyashiki, M. Sato and K. Takeda: Tetsu-to-Hagané, 96 (2010), 683. 